\title{
EPIMORPHISMS IN THE CATEGORY OF ABELIAN l-GROUPS
}

\author{
F. D. PEDERSEN ${ }^{1}$
}

ABSTRACT. By choosing the mappings for a category of $l$-groups carefully, some significant categorical results are obtainable. The intent of this paper is to investigate the reasons why epimorphisms in the category of $l$-homomorphisms between abelian $l$-groups are not "onto" functions.

Introduction. It was observed by D. Topping [5] that the category of all $l$-homomorphisms between $l$-groups contains no injectives. In view of this result, $\mathrm{P}$. Conrad [2] showed that in a more restrictive category (defined by N. Popa, 1965) the essentially closed l-groups are the injectives. Thus, by choosing the category carefully, some significant categorical results are obtainable. The intent of this paper is to investigate the reasons why epimorphisms in the category of $l$-homomorphisms between abelian l-groups are not "onto" functions. The definitions for the categorical notions are taken from Freyd, Abelian categories. $\$ 1$ contains definitions, examples, and some results concerning epimorphisms. $\$ 2$ discusses the relationship between epimorphisms and archimedean extensions. $\$ 3$ considers an extension of an $l$ group $G$ to another $l$-group $H$ so that the extended epimorphism to $H$ is an onto function. As a consequence of this extendability, certain types of $l$ groups are shown to have the property that all epimorphisms are onto functions.

1. For clarity of the exposition, the terms $l$-homomorphisms and $l$-isomorphisms are used when referring to functions from the theory of l-groups; morphisms, monomorphisms and epimorphisms are used when referring to functions from the category.

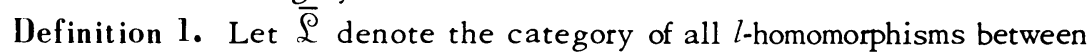 abelian l-groups.

Definition 2. A monomorphism $f \in \bar{\complement}$ is a morphism $f: G \rightarrow 4$ such that if $g: L \rightarrow G, h: L \rightarrow G$ and $f \circ g=f \circ h$, then $h=g$.

Definition 3. An epimorphism $f \in \overline{\mathcal{Q}}$ is a morphism $f: G \rightarrow: H$ such that if $g: H \rightarrow L, h: H \rightarrow L$ and $h \circ f=g \circ f$, then $h=g$.

Definition 4. An isomorphism $f \in \overline{\mathscr{L}}$ is a morphism $f: G \rightarrow H$ such that

Received by the editors July 22, 1974 and, in revised form, September 25, 1974.

AMS (MOS) subject classifications (1970). Primary 06A75; Secondary 06A60, $18 \mathrm{~A} 20$.

${ }^{1}$ The opportunity to discuss this subject with H. J. Biesterfeldt in seminar contributed greatly to the completion of this paper. 
there exists $g: H \rightarrow G$ such that $g \circ f=i_{G}$.

The reader can easily verify that the classes in question are categories; monomorphisms are one to one $l$-homomorphisms, and isomorphisms are $l$-isomorphisms. If $f \in \overline{\mathscr{Q}}$ and $f$ is a monomorphism which is also an onto l-homomorphism, then $f$ is an isomorphism. If $f \in \overline{\mathscr{Q}}$, then the first isomorphism theorem is true; i.e., if $f(G)$ denotes the image of $G$ in $H$, then $G / k(f)$ is l-isomorphic to $f(G)$ [4, Theorem 7, Chapter 2].

The following two examples illustrate that, in general,. epimorphisms are not onto l-homomorphisms and that this may or may not depend on the orders involved.

Example 1. This is an example of an epimorphism $f \in \bar{\complement}$ which is not onto as an $l$-homomorphism. It is also an epimorphism in the category of homomorphisms between torsion free groups. Let $G=2 I$ and $H=I$ where $I$ is the set of integers with their natural order. Let $f: G \rightarrow H$ by $f(x)=x$. Consider $g: H \rightarrow L$ and $h: H \rightarrow L$ such that $g \circ f=b \circ f \circ g(2)=b(2)=$ $2 h(1)=2 g(1)$. Thus, $2(g(1)-h(1))=0$. Since $L$ is torsion free, $g(1)=h(1)$, and $g=h$, since the values of $g(1)$ and $h(1)$ determine $g$ and $h$.

Example 2. This is an example of an epimorphism $f \in \overline{\mathscr{L}}$ which is not an onto $l$-homomorphism and is also not an epimorphism in the category of homomorphisms between torsion free groups. Let $G=\{m \vee 2: m \in I\}$ and $H$ $=\{n+m \vee 2: n, m \in I\}$. Use addition and the natural order on both $G$ and $H$. Define $f: G \rightarrow H$ by $f(m \vee 2)=m \vee 2$. Let $g: H \rightarrow L$ and $h: H \rightarrow L$ such that $g \circ f=h \circ f$. Since $H$ is archimedean, $g(x)=0$ for some $x>0$ implies that $g$ is the zero function. Therefore, we may assume that $g$ and $h$ are monomorphisms. Without loss of generality we may also assume that $g(H)$ and $h(H)$ are subgroups of the real numbers. Using [4, Proposition 2, Chapter 4], it follows that $g$ and $h$ are of the form $r x$ for some real number $r$. Since $g$ and $h$ agree on $\sqrt{ } 2$, they are equal. To see that $f$ is not an epimorphism in the category of homomorphisms between torsion free groups, define $h$ and $g$ as follows:

$$
g(n+m \sqrt{ } 2)=n, \quad h(n+m \sqrt{ } 2)=0 .
$$

Note that $g$ does not preserve order.

The following propositions point out a few facts about epimorphisms, some of which will be used in later sections of this paper.

Proposition 1. If $f \in \overline{\mathcal{L}}$ and $f$ is an epimorphism from $G$ to $H$, then the convex bull of $f(G)$ is equal to $H$.

Proof. Denote the convex hull by $[f(G)]$. Let $a$ be the natural l-homomorphism from $H$ to $H /[f(G)]$. Let $\phi$ be the zero function from it to $H /[f(G)] . \phi \circ f=\alpha \circ f$ which implies that $\phi=\alpha$ or that $H=[f(G)]$.

Proposition 2. If $f$ is an epimorphism from $G$ to $H, f \in \overline{\mathcal{Q}}$, and $H=$ 
$H_{1} \boxplus H_{2}$, then the composition of $f$ and either projection map is an epimorphism from $G$ to $H_{i}$.

Proposition 3. If $f \in \bar{\complement}$ is an epimorphism from $G$ to $H$ and $G=G_{1} \boxplus$ $G_{2}$, then $H=\left[f\left(G_{1}\right)\right] \boxplus\left[f\left(G_{2}\right)\right]$.

Proof. Let $0 \leq x \in\left[f\left(G_{1}\right)\right] \cap\left[f\left(G_{2}\right)\right]$ Since $f\left(G^{+}\right)=f(G)^{+}$, there exists $0<z, y$ such that $f(z) \in f\left(G_{1}\right)^{+}$and $f(y) \in f\left(G_{2}\right)^{+}$and $x \leq f(z), f(y)$. Thus, $f(z) \wedge f(y) \geq x$. Therefore, $f(z \wedge y) \geq x \geq 0$ which implies that $x=0$. Since $\left[f\left(G_{1}\right)\right] \boxplus\left[f\left(G_{2}\right)\right]$ is a convex l-subgroup of $H$ which contains $f(G), H=$ $\left[f\left(G_{1}\right)\right] \boxplus\left[f\left(G_{2}\right)\right]$.

Proposition 4. If $D$ is the divisible closure of the l-group $G$ and $D$ has the natural order extension from the order on $G$, then the identity from $G$ to $D$ is an epimorphism.

Proposition 5. If $f$ is an epimorphism from $G$ to $H$, and $D$ and $D^{\prime}$ are the divisible closures of $G$ and $H$, respectively, then there exists $g: D \rightarrow$ $D^{\prime}$ such that $g \mid G=f$.

Proof. Define $g(x)=f(n x) / n$ where $n$ is the least positive integer such that $n x \in G . g$ is well defined since $G$ is torsion free. If $y \in G$, then $g(y)$ $=f(y)$. Let $g(x+y)=f(n(x+y)) / n, g(x)=f(m x) / m$, and $g(y)=f(k y) / k$. Let $s=1 . c . m .\{n, m, k\}$. Since $f$ is a homomorphism it follows that $s f(n(x+y)) / n$ $=f(s(x+y)), \quad s f(m x) / m=f(s x), \quad s f(k y) / k=f(s y)$. Thus, $s g(x+y)=s g(x)$ $+s g(y)$ or $s(g(x+y)-g(x)-g(y))=0$. Since $D^{\prime}$ is torsion free, we have that $g(x+y)=g(x)+g(y)$. The order preserving properties of $g$ follow in a similar manner.

2. In order to obtain some connection between the order on $H$ and the l-subgroup $f(G)$ where $f$ is an epimorphism from $G$ to $H$, we consider the archimedean classes of $H$.

Definition 5. For two positive elements $x$ and $y, x$ is said to be $a$ equivalent to $y$ (denoted by $x \sim y$ ) if there exist two positive integers $n$ and $m$ such that $x \leq n y$ and $y \leq m x$.

Definition 6. An $l$-subgroup $G$ of $H$ is said to be $a$-equivalent to $H$ if for all $0<x \in H$ there exists $0<y \in G$ such that $x \sim y$.

From a paper by P. Conrad [1], it is noted that if $f(G)$ is an a-equivalent $l$-subgroup of $H$, then there exists an $l$-isomorphism $\sigma$ from the lattice of all convex $l$-subgroups of $f(G)$ onto the lattice of all convex $l$-subgroups of $H$.

Theorem l. If $f: G \rightarrow H$ is an epimorphism, $f \in \overline{\mathcal{L}}$, and if $f$ is an epimorphism in the category of homomorphisms between torsion free groups, then $H$ is an a-extension of $f(G)$. 
Proof. Suppose that $H$ is not an $a$-extension of $f(G)$. Then there exists a positive $x$ in $H$ such that no $y$ in $f(G)$ is $a$-equivalent to $x$. Let $D$ denote the divisible hull of $H$. Consider $f^{\prime}=i \circ f$ where $i$ is the identity from $H$ to $D . f^{\prime}$ is an epimorphism in the category of homomorphisms between torsion free groups. Consider $D$ as a vector space over the rationals. Let $S_{x}$ denote the subspace of $D$ generated by $x_{\bullet}$. Let $\overline{f^{\prime}(G)}$ denote the subspace of $D$ generated by $f^{\prime}(G)$. Suppose that $S_{x} \cap \overline{f^{\prime}(G)}$ is not the zero subspace and let $z$ be a nonzero element in $S_{x} \cap \frac{x}{f^{\prime}(G)} . \quad b z=a x$ for $a$ and $b$ integers. $d z=y$ for some $y \in f^{\prime}(G)$ where $d$ is an integer. Thus, $d a x=b y$ which contradicts our choice of $x$. Choose a basis for $\overline{f^{\prime}(G)}$ and extend it to $\overline{f^{\prime}(G)}+S_{x}$ with the addition of $x$. Then extend this basis for $\overline{f^{\prime}(G)}+S_{x}$ to the space $D$. Let $g$ be the homomorphism which maps $x$ to zero and $y$ $\rightarrow y$ for all basis elements $y \neq x$. $g$ will agree with the identity homomorphism on $f^{\prime}(G)$. This is a contradiction to $f^{\prime}$ being an epimorphism.

Definition 7. An epimorphism $g: A \rightarrow B$ is an a-epimorphism if $g$ is also an epimorphism in the category of homomorphisms between torsion free groups.

Note that Theorem 1 implies that for any a-epimorphism $g: A \rightarrow B, g \in$ $\overline{\mathcal{L}}, B$ is an archimedean extension of $g(A)$.

3. Throughout this section all the morphisms will be from $\overline{\mathcal{L}}$.

Definition 8. $(B, \alpha)$ is a completion of $A$ if $\alpha: A \rightarrow B$ is both a monomorphism and an $a$-epimorphism.

Definition 9. $(\hat{A}, \alpha)$ is an epicompletion of $A$ if

(i) $(\hat{A}, a)$ is a completion of $A$,

(ii) for all $a$-epimorphisms $f: A \rightarrow B$, there exists a completion $(\bar{B}, \delta)$ of $B$ and an "onto" morphism $\hat{f}: \hat{A} \rightarrow \bar{B}$ such that the following diagram commutes:

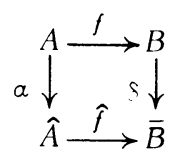

Definition 10. An l-group $G$ is epicomplete if all $a$-epimorphisms $f$ : $G \rightarrow H$ are onto functions.

Proposition 6. If $(\hat{\hat{A}}, \beta)$ is an epicompletion of $(\hat{A}, \alpha)$, then $(\hat{\hat{A}}, \beta \circ \alpha)$ is an epicompletion of $A$.

Proof. Since $\hat{f}$ is onto, $\hat{f}$ is an a-epimorphism, and can be extended to $\hat{\hat{f}}$.

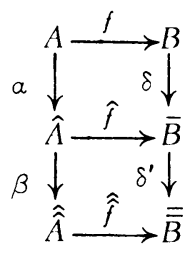


Proposition 7. In the following diagram, $\widehat{\beta \circ \alpha}=\pi \circ \beta$.



Proposition 8. $\hat{A} \simeq \hat{\hat{A}}$.

Proof. From the diagram and conclusion of Proposition 7, it follows that $\pi$ is onto since $\beta \circ \alpha$ is onto. Therefore $\pi$ is an isomorphism. Suppose there exists $g \in \hat{\hat{A}} \backslash \beta(\hat{A})$. Then there exists $z \in \hat{A}$ such that $\pi \circ \beta(z)=\pi(g)$. Since $\pi$ is a monomorphism, $\beta(z)=g$. This contradicts our choice of $g$. Therefore, $\beta$ is onto and $\hat{A} \simeq \hat{\hat{A}}$.

Proposition 9. If $(\hat{A}, \alpha)$ is an epicompletion of $A$, then any a-epimorphism $f: \hat{A} \rightarrow C$ is an onto function.

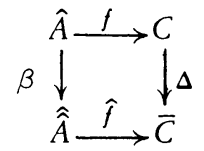

Proof. Let $c \in C$. There exists $g \in \hat{A}$ such that $\hat{f} \circ \beta(g)=\Delta(c)$. Since the diagram commutes, $\Delta \circ f(g)=\Delta(c) . \Delta$ is a monomorphism; thus, $f(g)=c$.

The previous material presented in this section has not considered the question as to whether $\hat{A}$ will exist for a given l-group $A$. A partial answer to this question will be stated in Theorem 2. The existence of epicomplete l-groups is established in Corollary 2 to Theorem 2. Needed for the proof of Theorem 2 is a result of P. Conrad's which is given below along with its accompanying diagram.

Theorem 4.1 [1]. Suppose that $G$ is a divisible abelian l-group, $\Delta$ is a plenary subset of $\Gamma_{1}(G)$ and $V\left(\Delta, R_{\delta}\right)=V$. Then there exists a fixed l-isomorphism $\phi$ of $G$ into $V$ such that if $G^{\prime}$ is any divisible a-extension of $G$, then $\phi$ can be extended to an l-isomorphism of $G^{\prime}$ into $V$.

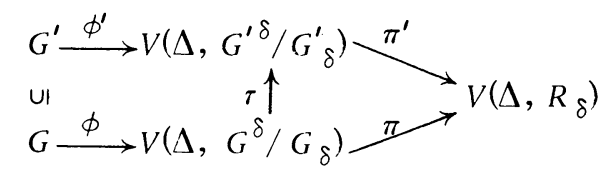

Theorem 2. If $f: G \rightarrow H$ is an a-epimorphism, then there exist representations for $G$ and $H, V\left(\triangle: R_{\delta}\right)$ and $V\left(\Delta, R_{\delta}\right)$, respectively $(\alpha$ and $\beta$ are embedding maps which go with the choice of $\Delta$ ' and $\Lambda$ ), and an "onto" morphism $\hat{f}$ so that the following diagram commutes:




Proof. Using Proposition 5, assume that $G$ and $H$ are divisible. Since the construction of the embedding for a divisible abelian l-group into a $V\left(\Delta, R_{\delta}\right)$ is to be used explicitly in this proof, a preliminary outline is given. A plenary subset $\Lambda$ is chosen from the root system $\Gamma_{1}(G) . \Gamma_{1}(G)$ is the collection of all regular $l$-ideals of $G$. A choice of a Banachewski function $\pi$ is used to construct the $l$-isomorphism. The mapping $\pi$ is a mapping of all subspaces of $G$ into itself such that for all subspaces $A$ and $B$ of $G$, (i) $G=A \oplus \pi(A)$, and (ii) if $A \subseteq B$, then $\pi(B) \subseteq \pi(A)$. The embedding map $\phi$ from $G$ to $V\left(\Delta, G^{\delta} / G_{\delta}\right)$ is defined by

$$
\phi(x)_{\delta}=x_{\delta}+G_{\delta} \text { where } x=x_{\delta}+c_{\delta}, x_{\delta} \in G^{\delta} \text { and } c_{\delta} \in \pi\left(G^{\delta}\right) \text {. }
$$

The extension from $V\left(\Lambda, G^{\delta} / G_{\delta}\right)$ to $V\left(\Lambda, R_{\delta}\right)$ is made by using the existence of an $o$-isomorphism from $G^{\delta} / G_{\delta}$ to a subgroup of the real numbers.

The proof of Theorem 2 will be reduced in the following way. According to the diagram given with 4.1 , let $f(G)=G$ and $H=G^{\prime}$. Then the following diagram is commutative:

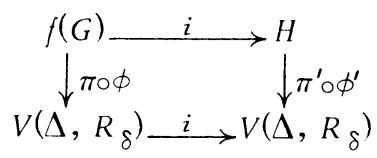

The existence of $\hat{f}$ now reduces to exhibiting the existence of $f^{\prime}, \alpha, \Delta^{\prime}$, and $\Delta$ so as to complete the following diagram:

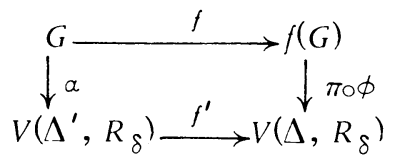

The choice of $\Delta$ comes later, and then Theorem 4.1 will give $\pi \circ \phi$. So the problem reduces to finding an $f^{\prime}$ which will be onto.

To further reduce the proof, let $\gamma$ be the $l$-isomorphism from $G / K$ to $f(G)$ where $K$ is the kernel of $f$ and $\gamma(x+K)=f(x)$. The composition of the natural homomorphism $\theta$ from $G$ to $G / K$ with the function $\gamma$ will equal $f$. Therefore, we may consider the following diagram:

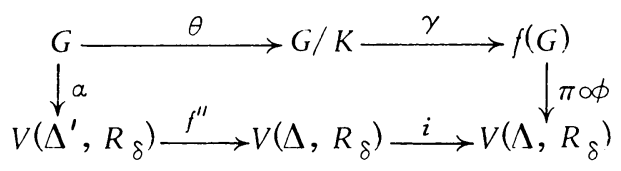

Now, with the choice of $\Delta, \pi \circ \phi$ will be given by Theorem 4.1, and we need only exhibit an $f^{\prime \prime}$ which is onto.

Choose $\Lambda^{\prime}$, a plenary set for $G$, such that $g \notin K$ implies that there exists a value $G_{\delta}$ of $g$ in $\Delta^{\prime}$ such that $G \supseteq K$. Let $\bar{\Delta}=\left\{G_{\delta} \in \Lambda^{\prime}: G_{\delta} \supseteq K\right\}$. Then, $\Delta=\left\{G_{\delta} / K: G_{\delta} \in \bar{\Delta}\right\}$ is a plenary set for $G / K$, and $\bar{\Delta}$ is, by construction, an upper class of $\Lambda^{\prime}$. Moreover, $K=\{g \in G: g$ does not have a value in $\bar{\Lambda}\}$. 
Let $\alpha^{\prime}: G \rightarrow V\left(\Lambda^{\prime}, G^{\delta}, G_{\delta}\right)$ be the embedding function constructed from the Banachewski function, $\pi$. Define $\tau^{\prime}: G / K \rightarrow V\left(\Delta,\left(G^{\delta} / K\right) /\left(G_{\delta} / K\right)\right)$ by $\tau^{\prime}(g+K)_{\delta}=\left(g_{\delta}+K\right)+G_{\delta} / K$. Note that $\tau^{\prime}$ is an embedding since

$$
G / K=\left(G^{\delta} \oplus \pi\left(G^{\delta}\right)\right) / K \simeq G^{\delta} / K \oplus \pi\left(G^{\delta}\right) .
$$

Define $g: V\left(\Lambda^{0}, G^{\delta} / G_{\delta}\right) \rightarrow V\left(\Lambda,\left(G^{\delta} / K\right) /\left(G_{\delta^{\prime}} K\right)\right)$ by $g(v)_{\delta}=\left(g_{\delta}+K\right)+G_{\delta^{\prime}} K$ where $v_{\delta}=g_{\delta}+G_{\delta}$ for each $\delta \in \Delta$. For each coordinate $\delta \in \Lambda$, this is the natural mapping of $G^{\delta} / G_{\delta}$ onto $\left(G^{\delta / K} /\left(G_{\delta} / K\right)\right.$. Thus, $g$ is an l-homomorphism, and $\tau^{\prime} \circ \theta=g \circ a^{\circ}$ 。

Let $h_{\delta}$ denote the natural $o$-isomorphism between $G^{\delta} / G_{\delta}$ and $\left(G^{\delta} / K\right) /\left(G_{\delta}^{\prime} K\right)$. Let $t_{1}$ denote an $o$-isomorphism between $G^{\delta} / G_{\delta}$ and $R_{\delta}$. The composition $t_{1} \circ h_{\delta}^{-1}$ is an o-isomorphism from $\left(G^{\delta} / K\right) /\left(G_{\delta} / K\right)$ to $R_{\delta}$. Let $\tau_{2}=b_{\delta}^{-1} \circ t_{1}$, and the following diagram commutes:

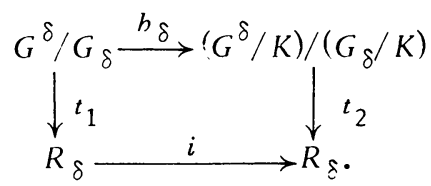

The function $f^{\prime \prime}$ is constructed in the obvious manner using the above diagram. Furthermore, $f^{\prime \prime}$ is onto. Thus, the existence of $\hat{f}$ has been established.

Corollary 1. If $G$ is an l-group and $\Delta$ is a plenary set for $G$ such that the embedding map $\pi$ of $G$ into $V\left(\Lambda, R_{\delta}\right)$ is an a-epimorphism, then there exists an epicompletion of $G$.

Corollary 2. $V\left(\Delta, R_{\delta}\right)$ is an epicomplete l-group.

\section{REFERENCES}

1. P. F. Conrad, Archimedean extensions of lattice-ordered groups, J. Indian Math. Soc. 30 (1966), 131-160 (1967). MR $37 \# 118$.

2. The essential closure of an Archimedean lattice-ordered group, Duke Math. J. 38 (1971), 151-160. MR 43 \#3190.

3. P. Conrad, J. Harvey and C. Holland, The Hahn embedding theorem for abelian lattice-ordered groups, Trans. Amer. Math. Soc. 108 (1963), 143-169. MR 27 \#1519.

4. L. Fuchs, Partially ordered algebraic systems, Pergamon Press, New York; Addison-Wesley, Reading, Mass., 1963. MR 30 \#2090.

5. D. M. Topping, Some homological pathology in vector lattices, Canad. J. Math. 17 (1965), 411-428. MR $30 \# 4700$.

DEPARTMENT OF MATHEMATICS, SOURTHERN ILLINOIS UNIVERSITY, CARBONDALE, ILLINOIS 62901

Current address: Department of Mathematics, University of Houston, Houston, Texas 77004 